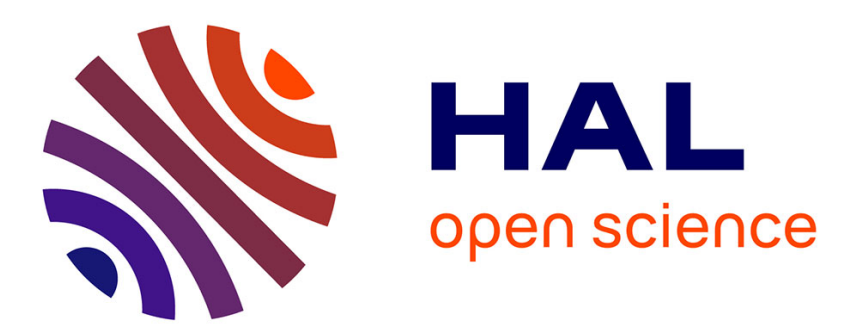

\title{
On Generalized Homogenization of Linear Quadrotor Controller
}

\author{
Siyuan Wang, Andrey Polyakov, Gang Zheng
}

\section{To cite this version:}

Siyuan Wang, Andrey Polyakov, Gang Zheng. On Generalized Homogenization of Linear Quadrotor Controller. IEEE International Conference on Robotics and Automation (ICRA)., May 2020, Paris, France. hal-02877399

\author{
HAL Id: hal-02877399 \\ https://hal.inria.fr/hal-02877399
}

Submitted on 22 Jun 2020

HAL is a multi-disciplinary open access archive for the deposit and dissemination of scientific research documents, whether they are published or not. The documents may come from teaching and research institutions in France or abroad, or from public or private research centers.
L'archive ouverte pluridisciplinaire HAL, est destinée au dépôt et à la diffusion de documents scientifiques de niveau recherche, publiés ou non, émanant des établissements d'enseignement et de recherche français ou étrangers, des laboratoires publics ou privés. 


\title{
On Generalized Homogenization of Linear Quadrotor Controller
}

\author{
Siyuan Wang, Andrey Polyakov, Gang Zheng
}

\begin{abstract}
A novel scheme for an "upgrade" of a linear control algorithm to a non-linear one is developed based on the concepts of a generalized homogeneity and an implicit homogeneous feedback design. Some tuning rules for a guaranteed improvement of a regulation quality are proposed. Theoretical results are confirmed by real experiments with the quadrotor QDrone of Quanser ${ }^{T M}$.
\end{abstract}

\section{INTRODUCTION}

Homogeneity is a kind of symmetry when an object (a function, a set, etc.) remains invariant with respect to a class of transformations, called dilations. For example, a symmetry of a function $f$ with respect to the uniform dilation of its argument $x \rightarrow e^{s} x$, where $s \in \mathbb{R}$ is the scaling parameter, is known as standard homogeneity. Any standard homogeneous function $f$ is characterized as follows $f\left(e^{s} x\right)=e^{s \nu} f(x)$, where a constant $\nu \in \mathbb{R}$ is called homogeneity degree. If we change the dilation rule then another (a generalized) homogeneity can also be defined [1], [2]. In this paper we deal mainly with the linear dilation [3] given by $x \rightarrow e^{G_{\mathbf{d}} s} x$, where $x \in \mathbb{R}^{n}, s \in \mathbb{R}$ and $G_{\mathbf{d}} \in \mathbb{R}^{n \times n}$ is an anti-Hurwitz matrix.

All linear and a lot of essentially nonlinear models of mathematical physics and mechanics are homogeneous (symmetric) in a generalized sense [4], [5]. Homogeneous models are utilized as local approximations of control systems [1], [6] if, for example, linearization is too conservative, noninformative or simply impossible. Many methods of both linear and nonlinear control theory can be applied for analysis and design of homogeneous control systems (see [1], [7], [2], [8], [9], [3], [10] and references therein). Homogeneity allows faster convergences [8], [11] and improves robustness properties of the system [6], [9].

Quality of any control system is estimated by many quantitative indexes (see e.g. [12], [13], [14]), which reflects control precision, robustness of the closed-loop system with respect to disturbances, energetic effectiveness, etc. A welltuned linear controller, such as PID (Proportional-IntegralDifferential) algorithm, guarantees a good enough control quality in many practical cases [12]. However, the theory of linear control systems reaches its peak and the further improvement of control performance using the same linear strategy looks impossible. Being a certain relaxation of linearity, the homogeneity could provide additional tools for

The authors acknowledge the support of CSC Grant, CPER DATA and ANR DIGISLID, ANR-18-CE40-0008. The second author also acknowledges the support of the Government of Russian Federation (Grant 08-08).

S. Wang, A. Polyakov and G. Zheng are with Inria Lille-Nord Europe, 40 av. Halley, Villeneuve d'Ascq, 59650, France $\{$ Siyuan. Wang (Andrey.Polyakov, Gang. Zheng) @inria.fr\} A. Polyakov is also with ITMO University, Saint-Petersburg, Russia. improvement of control quality. The main goal of the paper is to develop a scheme for an "upgrade" of a linear controller to a generalized homogeneous nonlinear one with a guaranteed improvement of regulation precision and to demonstrate its efficiency on real experiments.

Notation: $\mathbb{R}$ is the set of real numbers, $\mathbb{R}_{+}=\{x \in \mathbb{R}: x \geq$ $0\} ;\|\cdot\|$ is a norm in $\mathbb{R}^{n} ; \operatorname{diag}\left\{\lambda_{i}\right\}_{i=1}^{n}$ is the diagonal matrix with elements $\lambda_{i} ; P \succ 0(\prec 0, \succeq 0, \preceq 0)$ for $P \in \mathbb{R}^{n \times n}$ means that the matrix $P$ is symmetric and positive (negative) definite (semi definite); $\lambda_{\min }(P)$ and $\lambda_{\max }(P)$ represent the minimal and maximal eigenvalue of a matrix $P=P^{\top}$; for $P \succeq 0$ the square root of $P$ is a matrix $M=P^{\frac{1}{2}}$ such that $M^{2}=P$; a continuous function $\sigma: \mathbb{R}_{+} \rightarrow \mathbb{R}_{+}$belongs to the class $\mathcal{K}$ if it is monotone increasing and $\sigma(0)=0$.

\section{Preliminaries}

\section{A. Generalized Homogeneity}

As explained in Section I, the homogeneity is a symmetry of an object (e.g. a function) with respect to a group of transformations, called dilations. In the general case, instead of the uniform dilation of an argument $x \rightarrow e^{s} x, x \in \mathbb{R}^{n}, s \in$ $\mathbb{R}$ we can consider a non-uniform one

$$
x \rightarrow \mathbf{d}(s), \quad x \in \mathbb{R}^{n}, s \in \mathbb{R}
$$

where $\mathbf{d}(s) \in \mathbb{R}^{n \times n}$ and $s \in \mathbb{R}$ is a parameter of the dilation. To be a dilation, the family of operators $\mathbf{d}(s)$ must satisfy some restrictions [15] like: 1) $\mathbf{d}(0) x=x$;2) $\|\mathbf{d}(s) x\| \rightarrow 0$ as $s \rightarrow-\infty$; 3) $\|\mathbf{d}(s) x\| \rightarrow+\infty$ as $s \rightarrow+\infty$, for $x \neq 0$.

In [16], [7], [2], the dilation $\mathbf{d}$ is suggested to be generated as a flow of a $C^{1}$ vector field $\nu: \mathbb{R}^{n} \rightarrow \mathbb{R}^{n}$. Such a dilation $\mathbf{d}(s)$ is known as geometric dilation [7], [2]. In this paper we deal only with the so-called linear geometric dilations, which require the vector field $\nu$ to be linear, i.e. $\frac{d}{d s} \mathbf{d}(s)=G_{\mathbf{d}} \mathbf{d}(s)$, where $G_{\mathbf{d}} \in \mathbb{R}^{n \times n}$ is an anti-Hurwitz matrix. The matrix $G_{\mathbf{d}}$ is called a generator of the dilation. The dilation $\mathrm{d}$ in this case is given by the matrix exponential

$$
\mathbf{d}(s):=e^{G_{\mathbf{d}} s}=\sum_{i=0}^{\infty} \frac{s^{i} G_{\mathbf{d}}^{i}}{i !} .
$$

The dilation $\mathbf{d}$ is said to be monotone if $\mathbf{d}(s)$ is a strong contraction for any $s<0$; or strictly monotone if $\exists \beta>0$ :

$$
\|\mathbf{d}(s)\|:=\sup _{x \neq 0} \frac{\|\mathbf{d}(s) x\|}{\|x\|} \leq e^{\beta s}, \quad \forall s \leq 0 .
$$

Monotonicity of dilation plays an important role for the investigations of homogeneous geometrical structures in $\mathbb{R}^{n}$ as well as for the analysis of homogeneous control systems.

In a finite dimensional space, any dilation is monotone and strictly monotone if a norm in $\mathbb{R}^{n}$ is properly selected. 
Theorem 2.1: [3] Let $\mathbf{d}$ be a dilation in the Euclidean space $\mathbb{R}^{n}$ with the inner product $\langle u, v\rangle:=u^{\top} P v$, where $u, v \in \mathbb{R}^{n}$, and $0 \prec P=P^{\top} \in \mathbb{R}^{n \times n}$ is a positive definite symmetric matrix. The dilation $\mathbf{d}$ is strictly monotone in $\mathbb{R}^{n}$ equipped with the norm $\|z\|=\sqrt{\langle z, z\rangle}$ if and only if the following linear matrix inequality holds

$$
P G_{\mathbf{d}}+G_{\mathbf{d}}^{\top} P \succ 0, \quad P \succ 0
$$

where $G_{\mathbf{d}} \in \mathbb{R}^{n}$ is the generator of the dilation $\mathbf{d}$.

A dilation allows a new norm-topology to be introduced using the so-called canonical homogeneous norm [17]. The function $\|\cdot\|_{\mathbf{d}}: \mathbb{R}^{n} \rightarrow[0,+\infty)$ defined as $\|0\|_{\mathbf{d}}=0$ and

$$
\|x\|_{\mathbf{d}}=e^{s_{x}}, \text { where } s_{x} \in \mathbb{R}:\left\|\mathbf{d}\left(-s_{x}\right) x\right\|=1,
$$

is called the canonical homogeneous norm.

Obviously, $\|\mathbf{d}(s) x\|_{\mathbf{d}}=e^{s}\|x\|_{\mathbf{d}}$ and $\|x\|_{\mathbf{d}}=\|-x\|_{\mathbf{d}}$ for any $x \in \mathbb{R}^{n}$ and any $s \in \mathbb{R}$. The homogeneous norm defined by (4) was called canonical since it is induced by a canonical norm $\|\cdot\|$ in $\mathbb{R}^{n}$ and $\|x\|_{\mathbf{d}}=1 \Leftrightarrow\|x\|=1$. Notice that for $\mathbf{d}(s)=e^{s}$ the canonical homogeneous norm $\|\cdot\|$ coincides with the norm $\|\cdot\|$ on the whole $\mathbb{R}^{n}$.

The monotonicity of the dilation group guarantees that the function $\|\cdot\|_{\mathbf{d}}$ is single-valued and continuous at the origin [3]. Moreover, if the norm in $\mathbb{R}^{n}$ is defined as $\|x\|=\sqrt{x^{\top} P x}$ with $P \in \mathbb{R}^{n \times n}$ satisfying (3) then $\|\cdot\|_{\mathbf{d}}$ is continuously differentiable outside the origin

$$
\frac{\partial\|x\|_{\mathbf{d}}}{\partial x}=\|x\|_{\mathbf{d}} \frac{x^{\top} \mathbf{d}^{\top}\left(-\ln \|x\|_{\mathbf{d}}\right) P \mathbf{d}\left(-\ln \|x\|_{\mathbf{d}}\right)}{x^{\top} \mathbf{d}^{\top}\left(-\ln \|x\|_{\mathbf{d}}\right) P G_{\mathbf{d}} \mathbf{d}\left(-\ln \|x\|_{\mathbf{d}}\right) x}, \quad x \neq 0 .
$$

It is well known (see e.g. [18]) that the norm $\|x\|=$ $\sqrt{x^{\top} P x}$ is a Lyapunov function for any stable linear system $\dot{x}=A x, A \in \mathbb{R}^{n \times n}$. It is expectable that the canonical homogeneous norm would define a Lyapunov function for a class of stable homogeneous systems.

\section{B. Homogeneous Systems}

Homogeneity (dilation symmetry) of the vector fields is given by the following definition, which, originally, has been proposed by L. Euler in 18th century for the uniform dilation. The generalized homogeneity is studied in [1], [2], [6], [10].

Definition 2.1: A vector field $f: \mathbb{R}^{n} \rightarrow \mathbb{R}^{n}$ is said to be $\mathrm{d}$-homogeneous of degree $\nu \in \mathbb{R}$ if

$$
f(\mathbf{d}(s) x)=e^{\nu s} \mathbf{d}(s) f(x) \quad \text { for } \quad s \in \mathbb{R}, \quad x \in \mathbb{R}^{n} .
$$

A lot of examples of $\mathbf{d}$-homogeneous nonlinear vector fields can be found in the literature (see Introduction). Obviously, any linear vector field $x \rightarrow A x, A \in \mathbb{R}^{n \times n}$ is homogeneous of the zero degree with respect to the uniform dilation $x \rightarrow$ $e^{s} x$. However, in some cases the linear vector field may be d-homogeneous of positive or negative degree dependently of a concrete dilation $\mathbf{d}$. The vector field $x \rightarrow A x$ with $A \in \mathbb{R}^{n \times n}$ and $x \in \mathbb{R}^{n}$ is d-homogeneous of degree $\nu \in \mathbb{R}$ if and only if [3]

$$
A G_{\mathbf{d}}=\left(\nu I+G_{\mathbf{d}}\right) A
$$

where $G_{\mathbf{d}} \in \mathbb{R}^{n \times n}$ is a generator of $\mathbf{d}$.

Homogeneity of a function (a vector field) is inherited by other mathematical object induced by this function such a derivatives or solutions of differential equations. If the right hand side of the following differential equation

$$
\dot{\xi}=f(\xi), \quad t>0, f: \mathbb{R}^{n} \rightarrow \mathbb{R}^{n}
$$

is $\mathbf{d}$-homogeneous of degree $\nu$ then

$$
x_{\mathbf{d}(s) x_{0}}(t)=\mathbf{d}(s) x_{x_{0}}\left(e^{\nu s} t\right), t>0
$$

where $x_{x_{0}}(t), t>0$ denotes a solution of (8) with the initial condition $x(0)=x_{0}$ (see e.g. [3]). The obtained symmetry of solution implies the fast (finite-time/fixed-time) convergence properties of the homogeneous systems dependently of the homogeneity degrees. The next proposition was originally proven for the weighted homogeneous systems in [11].

Proposition 2.1: [19] If the system (8) is d-homogeneous of degree $\nu \in \mathbb{R}$ and its origin is locally uniformly asymptotically stable then 1) for $\nu<0$ it is globally uniformly finite-time stable, i.e. there exists a $\mathrm{d}$-homogeneous settlingtime function $T: \mathbb{R}^{n} \rightarrow[0,+\infty)$ of degree 1 , which is locally bounded and continuous at 0 , such that $x_{x_{0}}(t)=0, \quad \forall t \geq$ $T\left(x_{0}\right)$; 2) for $\nu=0$ it is globally uniformly asymptotically stable; 3) for $\nu>0$ it is globally uniformly nearly fixed-time stable, i.e. $\forall r>0, \exists T=T(r)>0:\left\|x_{x_{0}}(t)\right\|<r, \forall t \geq T$, $\forall x_{0} \in \mathbb{R}^{n}$.

\section{Homogenization of LineAR Controller}

\section{A. Homogeneous Stabilization of Linear MIMO Systems}

Let us consider the linear control system

$$
\dot{x}=A x+B u(x), \quad t>0,
$$

where $x(t) \in \mathbb{R}^{n}$ is the system state, $u: \mathbb{R}^{n} \rightarrow \mathbb{R}^{m}$ is the feedback control, $A \in \mathbb{R}^{n \times n}, B \in \mathbb{R}^{n \times m}$ are system matrices.

Definition 3.1: A system (9) is said to be $\mathbf{d}-$ homogeneously stabilizable with degree $\mu \in \mathbb{R}$ if there exists a (locally or a globally bounded) feedback law $u: \mathbb{R}^{n} \rightarrow \mathbb{R}^{m}$ such that the closed-loop system is globally asymptotically stable and $\mathbf{d}$-homogeneous of degree $\mu$, where $\mathbf{d}$ is a dilation in $\mathbb{R}^{n}$.

In [20], it is shown that the system (9) can be homogeneously stabilized with a degree $\mu \neq 0$ if and only if the pair $\{A, B\}$ is controllable (or, equivalently, $\operatorname{rank}\left(B, A B, \ldots, A^{n-1} B\right)=n$. We refer the reader to [21] for more details about controllability of linear plants. The following theorem is the corollary of a more general theorem proven in [17] for evolution system in Hilbert spaces (see also [20] for more details about the finite dimensional case).

Theorem 3.1: If the pair $\{A, B\}$ is controllable and $\mu \in$ $\left[-1, k^{-1}\right]$, where $k \leq n: \operatorname{rank}\left(B, A B, \ldots, A^{k-1} B\right)=n$, then a homogeneously stabilizing control for (9) can always be selected in the form

$$
u(x)=K_{0} x+\|x\|_{\mathbf{d}}^{1+\mu} Y X^{-1} \mathbf{d}\left(-\ln \|x\|_{\mathbf{d}}\right) x
$$

with any $K_{0} \in \mathbb{R}^{n \times m}$ such that $A_{0}=A+B K_{0}$ is nilpotent, any dilation $\mathbf{d}$ generated by $G_{\mathbf{d}} \in \mathbb{R}^{n \times n}$ satisfying

$$
A_{0} G_{\mathbf{d}}=\left(G_{\mathbf{d}}+\mu I\right) A_{0}, \quad G_{\mathbf{d}} B=B
$$


and any $X \in \mathbb{R}^{n \times n}, Y \in \mathbb{R}^{m \times n}$ solving the following algebraic system

$$
\left\{\begin{array}{l}
X A_{0}^{\top}+A_{0} X+Y^{\top} B^{\top}+B Y+X G_{\mathbf{d}}^{\top}+G_{\mathbf{d}} X=0, \\
X G_{\mathbf{d}}^{\top}+G_{\mathbf{d}} X \succ 0, \quad X \succ 0,
\end{array}\right.
$$

where the canonical homogeneous norm $\|\cdot\|_{\mathbf{d}}$ is induced by the norm $\|x\|=\sqrt{x^{\top} X^{-1} x}$. Moreover, the canonical homogeneous norm is a Lyapunov function of the closedloop system (9), (10) and $\frac{d}{d t}\|x(t)\|_{\mathbf{d}}=-\|x(t)\|_{\mathbf{d}}^{1+\mu}$.

Notice that dependently of the parameter $\mu$ the proposed controller guarantees finite-time $(\mu<0)$ or nearly fixed-time $(\mu<0)$ stabilization of the system (see Proposition (2.1)).

The linear control theory uses an integral term in order to improve robustness properties of a proportional laws. This scheme works also for nonlinear controllers [22]. An integrator can be added to a homogeneous feedback [23].

Theorem 3.2: Let $K_{0} \in \mathbb{R}^{m \times n}$ be such that $A+B K_{0}$ is nilpotent and an anti-Hurwitz matrix $G_{\mathbf{d}} \in \mathbb{R}^{n \times n}$ satisfy (11). Let $X \in \mathbb{R}^{n \times n}$ and $Y \in \mathbb{R}^{m \times n}$ satisfy (12) then for any positive definite matrix $Q \in \mathbb{R}^{m \times m}$ the control law

$$
u(x)=K_{0} x+u_{h}(x)+\int_{0}^{t} u_{I}(x(s)) d s,
$$

with $u_{h}=\|x\|_{\mathbf{d}}^{1 / 2} Y X^{-1} z, u_{I}=\frac{-Q B^{\top} P z}{z^{\top} P G_{\mathbf{d}} z}, z=\mathbf{d}\left(-\ln \|x\|_{\mathbf{d}}\right) x$ stabilizes the origin of the system $\dot{x}=A x+B(u(x)+p)$, in a finite-time time for any constant vector $p \in \mathbb{R}^{m}$, where, as before, the canonical homogeneous norm $\|\cdot\|_{\mathbf{d}}$ is induced by the norm $\|x\|=\sqrt{x^{\top} X^{-1} x}$.

Notice that $0.5 \lambda_{\min }\left(P^{1 / 2} G_{\mathbf{d}} P^{-1 / 2}+P^{-1 / 2} G_{\mathbf{d}}^{\top} P^{1 / 2}\right) \leq$ $z^{\top} P G_{\mathbf{d}} z \leq 0.5 \lambda_{\max }\left(P^{1 / 2} G_{\mathbf{d}} P^{-1 / 2}+P^{-1 / 2} G_{\mathbf{d}}^{\top} P^{1 / 2}\right)$ for $z=\mathbf{d}\left(-\ln \|x\|_{\mathbf{d}}\right) x$ and, for practical reasons, the integral part of the above controller can be selected as $\tilde{u}_{I}=$ $-Q B^{\top} P \mathbf{d}\left(-\ln \|x\|_{\mathbf{d}}\right) x$ provided that the closed-loop system remains stable.

\section{B. From a linear feedback to a homogeneous control law}

The PID (Proportional-Integral-Derivative) controller is the most common linear feedback law for real physical control systems. In the previous sections we have shown that homogeneous systems may have faster convergence rates. In this section we study the question: Is it possible to upgrade an existing linear controller in order to make a closed-loop system locally or globally $\mathbf{d}$-homogeneous and to improve convergence properties of the system? A scheme of the upgrade must prevent a possible degradation of the control quality allowing only its improvement.

Let us consider again the linear system (9) and assume that some linear control law

$$
u_{\text {lin }}(x)=K_{\text {lin }} x, \quad K_{\text {lin }} \in \mathbb{R}^{m \times n}, \quad x \in \mathbb{R}^{n}
$$

is already designed.

Corollary 3.1: Let the pair $\{A, B\}$ be controllable and $K_{0} \in \mathbb{R}^{m \times n}$ be such that the matrix $A_{0}=A+B K_{0}$ is nilpotent and $K_{\text {lin }} \in \mathbb{R}^{m \times n}$ be such that the matrix $A+$ $B K_{\text {lin }}$ is Hurwitz. Let $G_{\mathbf{d}} \in \mathbb{R}^{n \times n}$ be a generator of the dilation $\mathbf{d}$ such that (11) holds for $\mu=-1$. If a matrix $P=$ $P^{\top} \in \mathbb{R}^{n \times n}$ satisfies the system of linear matrix inequalities

$$
\begin{aligned}
& \left(A+B K_{\text {lin }}\right)^{\top} P+P\left(A+B K_{\text {lin }}\right) \prec 0 \\
& G_{\mathbf{d}}^{\top} P+P G_{\mathbf{d}} \succ 0, \quad P \succ 0
\end{aligned}
$$

then the control $u$ given by (10) with $\mu=-1$ and $K=$ $K_{\text {lin }}-K_{0} \mathrm{~d}$-homogeneously stabilizes the origin of the system (9) in a finite-time, where $\|\cdot\|_{\mathrm{d}}$ is the canonical homogeneous norm induced by the norm $\|x\|=\sqrt{x^{\top} P x}$. Moreover, $u_{\text {lin }}(x)=u(x)$ for $x \in S=\left\{x \in \mathbb{R}^{n}:\|x\|=1\right\}$.

The latter corollary shows that if a linear controllable plant is exponentially stabilized by means of a linear feedback then it can also be homogeneously stabilized by means of the control (10) using the gains of the original linear controller. These two controllers are coincide on the unit sphere $x^{\top} P x=1$. Notice that the corresponding sphere can always be adjusted (if needed) by means of a variation of $P$ satisfying (14).

Let the saturation function $\mathrm{sat}_{a, b}: \mathbb{R}_{+} \rightarrow \mathbb{R}_{+}$be given by

$$
\text { sat }_{a, b}(\rho)=\left\{\begin{array}{cc}
b & \text { if } \quad \rho \geq b, \\
\rho & \text { if } a<\rho<b, \\
a & \text { if } \quad \rho<a,
\end{array} \quad \rho \in \mathbb{R}_{+} .\right.
$$

Let us consider the control law

$$
u_{a, b}(x)=K_{0} x+K \mathbf{d}\left(-\ln \operatorname{sat}_{a, b}\left(\|x\|_{\mathbf{d}}\right)\right) x,
$$

where $\mathbf{d},\|x\|_{\mathbf{d}}, K_{0}$ and $K=K_{\text {lin }}-K_{0}$ are defined in Corollary 3.1. From (15) we conclude that

$u_{1,1}(x)=K_{\text {lin }} x$ and $u_{0,+\infty}(x)=K_{0} x+K \mathbf{d}\left(-\ln \|x\|_{\mathbf{d}}\right) x$.

In other words, the pair $a \in(0,1]$ and $b \in[1,+\infty)$ parameterize a family of nonlinear controllers which has the linear and homogeneous feedbacks as the limit cases.

Notice that for $b=1$ the controller (16) coincides with the linear controller outside the unit ball $x^{\top} P x>1$ and the gains of the linear controller are scaled by means of a dilation $\mathbf{d}$ only close to the origin, i.e. for $x^{\top} P x<1$.

The following scheme for an "upgrade" of linear control to nonlinear (locally homogeneous) one can be suggested:

- Select any $K_{0} \in \mathbb{R}^{m \times n}$ such that $A+B K_{0}$ is nilpotent.

- Find a symmetric matrix $P=P^{\top}$ satisfying the inequalities (14), which is required to define the canonical homogeneous norm $\|\cdot\|_{\mathbf{d}}$.

- Select $a=b=1$ (i.e. we start with a linear controller).

- Increase $b>1$ and decrease $\alpha<1$ while this improves a quality of the control system.

Theoretically, an improvement of control quality (e.g. faster transition) follows from Proposition 2.1. However, the proof is model-based, but any model of a system is just an approximation of the reality. In practice, a difference between a model of dynamics and a real motion of the system may not allow to realize all theoretical properties of the closed-loop system or, even more, it may imply a serious degradation of some performance indexes, which characterize the control quality. The tuning of parameters $a$ and $b$ suggested above is required to guarantee that the nonlinear control always has a quality which is never worse than the original linear one. It would allow a control engineer 
to prevent any possible degradation of the control quality during the nonlinear "upgrade" of a linear control system. In Section IV we will illustrate the presented scheme on a real experiment with a quadrotor.

Notice that if the gains of the linear controller are already optimally adjusted, then improvements provided by homogeneous controller could not be huge and the parameters $a$ and $b$ could, possibly, be close 1 in this case.

\section{On digital realization of implicit homogeneous feedback}

It is important to know whether the proposed nonlinear homogeneous algorithms remain stable in the case of their implementation in a digital controller.

Theorem 3.3: If all conditions of Theorem 3.1 are fulfilled, then for any fixed $r>0$ the closed $\mathbf{d}$-homogeneous ball $\|x\|_{\mathbf{d}}<r$ is a strictly positively invariant compact set ${ }^{1}$ of the closed-loop system (9) with the linear control

$$
u_{r}(x)=K_{0}+r^{1+\mu} K \mathbf{d}(-\ln r) x .
$$

We skip the proofs of all claims due to space limitations.

Now we assume that the value $\|x(t)\|_{\mathbf{d}}$ can be changed only in some sampled instances of time and let us show the corresponding linear switched feedback robustly stabilize the perturbed linear system.

Corollary 3.2: If 1) all conditions of Theorem 3.1 are fulfilled; 2) $\left\{t_{i}\right\}_{i=0}^{+\infty}$ is an arbitrary sequence of time instances such that $0=\bar{t}_{0}<t_{1}<t_{2}<\ldots$ and $\lim _{i \rightarrow+\infty} t_{i}=+\infty$; 3) $u$ is a linear switched control of the from

$$
u(x(t))=\left\|x\left(t_{i}\right)\right\|_{\mathbf{d}}^{1+\mu} K \mathbf{d}\left(-\ln \left\|x\left(t_{i}\right)\right\|_{\mathbf{d}}\right) x(t), t \in\left[t_{i}, t_{i+1}\right)
$$

then the closed-loop system (9) is globally uniformly asymptotically stable.

The linear switched control (18) is obtained from the nonlinear homogeneous one. It can be utilized, for example, in the case when the control system is already equipped with a linear (e.g. analog) controller allowing a dynamic change of feedback gains with some sampling period.

According to the above corollary, the proposed sampledtime realization of the implicit homogeneous controller guarantees asymptotic stabilization of the closed-loop system independently of the dwell time (a time between two samplings). However, without any assumption on the dwell-time we cannot estimate the convergence rate of this system. Obviously, if the dwell time tends to zero, the convergence rate tends to the rate of the original homogeneous system. For more details about approximation of the canonical homogeneous norm we refer the reader to [19].

\section{EXAMPLE: AN “UPGRADE" OF A LINEAR CONTROLLER FOR Q-DRONE QUANSER ${ }^{T M}$}

\section{A. Model Description}

The dynamic model of quadrotor has been well established in the previous research [24]. The dynamic model of quadrotor might be expressed in three different frames: the inertial, horizon and body frame, denoted by $I, H$ and $B$

\footnotetext{
${ }^{1} \mathrm{~A}$ set $\Omega$ is said to be a strictly positively invariant for a dynamical system if $x\left(t_{0}\right) \in \Omega \Rightarrow x(t) \in \int \Omega, t \geq t_{0}$, where $x$ denotes a solution $x$ of this system.
}

respectively. Frame $I$ and $B$ are depicted in Fig. 1 (left). The frame $H$ is illustrated in Fig. 1 (right). Precisely, the body frame is attached to the mass center of quadrotor. Based on the horizon frame, a rotation about the $y$ axis by roll angle $\phi$, followed by a rotation about the new interim $x$ axis by pitch angle $\theta$ establish the body frame.

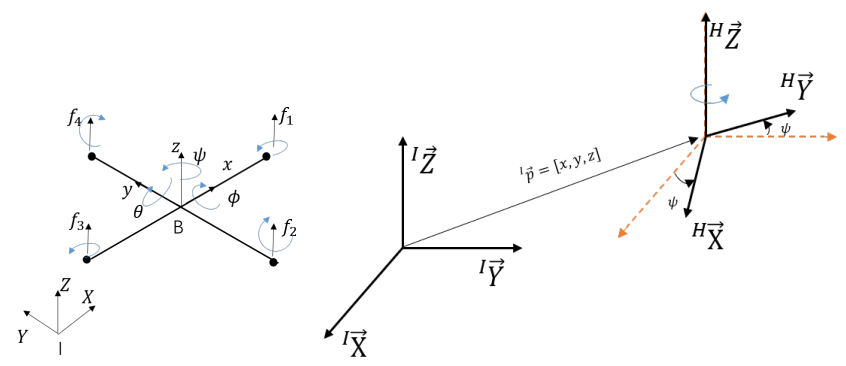

Fig. 1. Quadrotor coordinates and the horizon frame

In this paper, the hub forces, moments and Coriolis Force are considered as disturbance [24]. Therefore, the thrust force $\left(F_{T}\right)$ and the gravity $(\mathrm{mg})$ are two main forces to result in the quadrotor's motion. The thrust force is perpendicular to the quadrotor's main body plane (along the body frame $\mathrm{z}$ axis). The linear acceleration of quadrotor in different frames can be projected to the horizon frame by using roll $\phi$, pitch $\theta$ and yaw $\psi$, which is ${ }^{H} \vec{a}={ }^{H} R_{B}{ }^{B} \vec{a}-{ }^{H} R_{I}{ }^{I} \vec{g}$, where

${ }^{H} R_{B}=\left[\begin{array}{ccc}\cos \theta & \sin \theta \sin \phi & \sin \theta \cos \phi \\ 0 & \cos \phi & -\sin \phi \\ -\sin \theta & \cos \theta \sin \phi & \cos \theta \cos \phi\end{array}\right] ;{ }^{H} R_{I}=\left[\begin{array}{ccc}\cos \psi & -\sin \psi & 0 \\ \sin \psi & \cos \psi & 0 \\ 0 & 0 & 1\end{array}\right]$

Thus

${ }^{H}\left[\begin{array}{c}\ddot{x} \\ \ddot{y} \\ \ddot{z}\end{array}\right]=\left[\begin{array}{ccc}\cos \theta & \sin \theta \sin \phi & \sin \theta \cos \phi \\ 0 & \cos \phi & -\sin \phi \\ -\sin \theta & \cos \theta \sin \phi & \cos \theta \cos \phi\end{array}\right]^{B}\left[\begin{array}{l}0 \\ 0 \\ \frac{F_{T}}{m}\end{array}\right]-\left[\begin{array}{ccc}\cos \psi & \sin \psi & 0 \\ -\sin \psi & \cos \psi & 0 \\ 0 & 0 & 1\end{array}\right]^{B}\left[\begin{array}{l}0 \\ 0 \\ g\end{array}\right]$ which yields the following nonlinear model [24]:

$$
{ }^{H} \vec{a}=\left[\begin{array}{c}
\ddot{x} \\
\ddot{y} \\
\ddot{z}
\end{array}\right]=\left[\begin{array}{c}
\frac{F_{T}}{m} \sin \theta \cos \phi \\
-\frac{F_{T}}{m} \sin \phi \\
\frac{F_{T}}{m} \cos \theta \cos \phi-g
\end{array}\right]
$$

where $^{H}\left[\begin{array}{c}\ddot{x} \\ \ddot{y} \\ \ddot{z}\end{array}\right]$ represents the linear accelerations in horizon frame. The Euler equation $J \dot{\omega}+\omega \times J \omega=\tau$ leads to the following three angular motion equations [24]:

$$
\ddot{\phi}=\frac{1}{I_{x x}} \tau_{1} ; \quad \ddot{\theta}=\frac{1}{I_{y y}} \tau_{2} ; \quad \ddot{\psi}=\frac{1}{I_{z z}} \tau_{3}
$$

where $I_{x x}, I_{y y}$ and $I_{z z}$ are the principle roll, pitch and yaw moments of inertia. $\tau_{1}, \tau_{2}$ and $\tau_{3}$ are the corresponding applied torques in body frame. To demonstrate the proposed method of a homogeneous PID controller design, a linearized model of (19) and a linear PID controller provided by the manufacturer are required.

\section{B. Quanser platform}

The test platform of this paper is Quanser QDrone ${ }^{\mathrm{TM}}$ which is a midsize quadrotor equipped with a powerful onboard Intel Aero Compute Board. The hardware parameters of quadrotor (provided by the manufacturer) are $m=$ 
$1.07 \mathrm{~kg}$, the gravity $g=9.8 \mathrm{~m} / \mathrm{s}^{2}$, the motor distance $L_{\text {roll }}=$ $0.2136 \mathrm{~m}, L_{\text {pitch }}=0.1758 \mathrm{~m}$, the roll inertia $I_{x x}=6.85 \times$ $10^{-3} \mathrm{kgm}^{2}$, the pitch inertia $I_{y y}=6.62 \times 10^{-3} \mathrm{kgm}^{2}$, the yaw inertia $I_{z z}=1.29 \times 10^{-2} \mathrm{kgm}^{2}$, The thrust coefficient $k=1.93 \times 10^{-8} \frac{N}{R P M^{2}}$, the drag coefficient $c=0.26 \times$ $10^{-9} \frac{N m}{R P M^{2}}$. The position and linear velocity are measured by OptiTrack ${ }^{\mathrm{TM}}$ and computed on board respectively. The angle rate are measured by IMU and the attitude angle are computed from the measurement of IMU.

\section{Linear and homogeneous controllers}

A linearized model of (19) and (20) is given by:

$$
H \vec{a}=\left[\begin{array}{c}
\ddot{x} \\
\ddot{y} \\
\ddot{z}
\end{array}\right]=\left[\begin{array}{c}
\frac{F_{T}}{m} \sin \theta \cos \phi \\
-\frac{F_{T}}{m} \sin \phi \\
\frac{F_{T}}{m} \cos \theta \cos \phi-g
\end{array}\right] \approx\left[\begin{array}{c}
g \theta \\
-g \phi \\
\frac{F_{T}}{m}-g
\end{array}\right]
$$

where $F_{T}$ is assumed to be close to $m g$ and the angle $\theta, \phi$ are assumed to be close to 0 . Denoting $\zeta=$ $(x, y, \dot{x}, \dot{y}, \theta,-\phi, \dot{\theta},-\dot{\phi})^{\top}$ from (21) and (20) we derive

$$
\dot{\zeta}=A \zeta+\left(\begin{array}{l}
0 \\
0 \\
0 \\
B
\end{array}\right)\left(\begin{array}{l}
u_{2} \\
u_{3}
\end{array}\right), \quad \ddot{\psi}=\frac{u_{4}}{I_{z z}}, \quad \ddot{z}=\frac{u_{1}}{m}
$$

where $u_{1}=F_{T}-m g, u_{2}=\tau_{1}, u_{3}=\tau_{2}, u_{4}=\tau_{3}$

$$
A=\left(\begin{array}{cccc}
0 & E & 0 & 0 \\
0 & 0 & g E & 0 \\
0 & 0 & 0 & E \\
0 & 0 & 0 & 0
\end{array}\right), \quad E=\left(\begin{array}{ll}
1 & 0 \\
0 & 1
\end{array}\right), \quad B=\left(\begin{array}{cc}
\frac{1}{I_{y y}} & 0 \\
0 & \frac{1}{I_{x x}}
\end{array}\right) \text {. }
$$

The PID controllers provided by Quanser are of the following form:

$$
u_{1}=K_{z}\left(\begin{array}{c}
z \\
\dot{z}
\end{array}\right)+\int K_{4} z d t, \quad\left(\begin{array}{l}
u_{2} \\
u_{3}
\end{array}\right)=K_{\zeta} \zeta, \quad u_{4}=K_{\psi}\left(\begin{array}{c}
\psi \\
\dot{\psi}
\end{array}\right)
$$

with the parameters (provided by the manufacturer)

$$
\begin{aligned}
& K_{\psi}=\left[\begin{array}{ll}
-0.59 & 0.11
\end{array}\right] \quad K_{z}=\left[\begin{array}{ll}
-35 & -14
\end{array}\right], K_{I}=-4 \\
& K_{\zeta}=\left[\begin{array}{cccccccc}
-2.91 & 0 & -1.45 & 0 & -1.85 & 0 & -0.16 & 0 \\
0 & -3.53 & 0 & -1.76 & 0 & -2.25 & 0 & -0.20
\end{array}\right] \text {. }
\end{aligned}
$$

The same gains are utilized for homogeneous controllers of the form (10) and (13). Each linear subsystem in (22) is $\mathbf{d}$-homogeneous of degree -1 with respect to the dilations: $\mathbf{d}_{1}(s)=\operatorname{diag}\left\{e^{4 s} E, e^{3 s} E, e^{2 s} E, e^{1 s} E\right\}$ and $\mathbf{d}_{2}(s) \operatorname{diag}\left\{e^{2 s}, e^{s}\right\}, s \in \mathbb{R}$. Therefore, the linear terms $K_{0} x$ in (10) can be omitted.

In our test, the deduced nonlinear homogeneous controllers are implemented with the following parameters: $a_{\zeta}=$ $0.6, a_{\psi}=0.65$, and $a_{z}=0.3$ and $b_{\zeta}=b_{\psi}=b_{z}=1$.

\section{Experiment results}

In the experiment we compare Quanser's linear PID controller with the homogeneous PID controller obtained from the linear one by means of a generalized homogeneous dilation. The experiment consists in the sequential set-points tracking. The reference positions to be tracked are defined in initial frames as follows: $[x, y, z, \psi]=[0,0,0,0] \rightarrow$ $[0,0,0.4,0] \rightarrow[0.2,0,0.4,0] \rightarrow[0.2,0.2,0.4,0] \rightarrow$ $[0,0,0.4,0] \rightarrow[0,0,0.018,0]$.

Fig. 2 depicts the position tracking trajectory in $x, y, z$ and $\psi$ variables, respectively. It is easy to see that the nonlinear
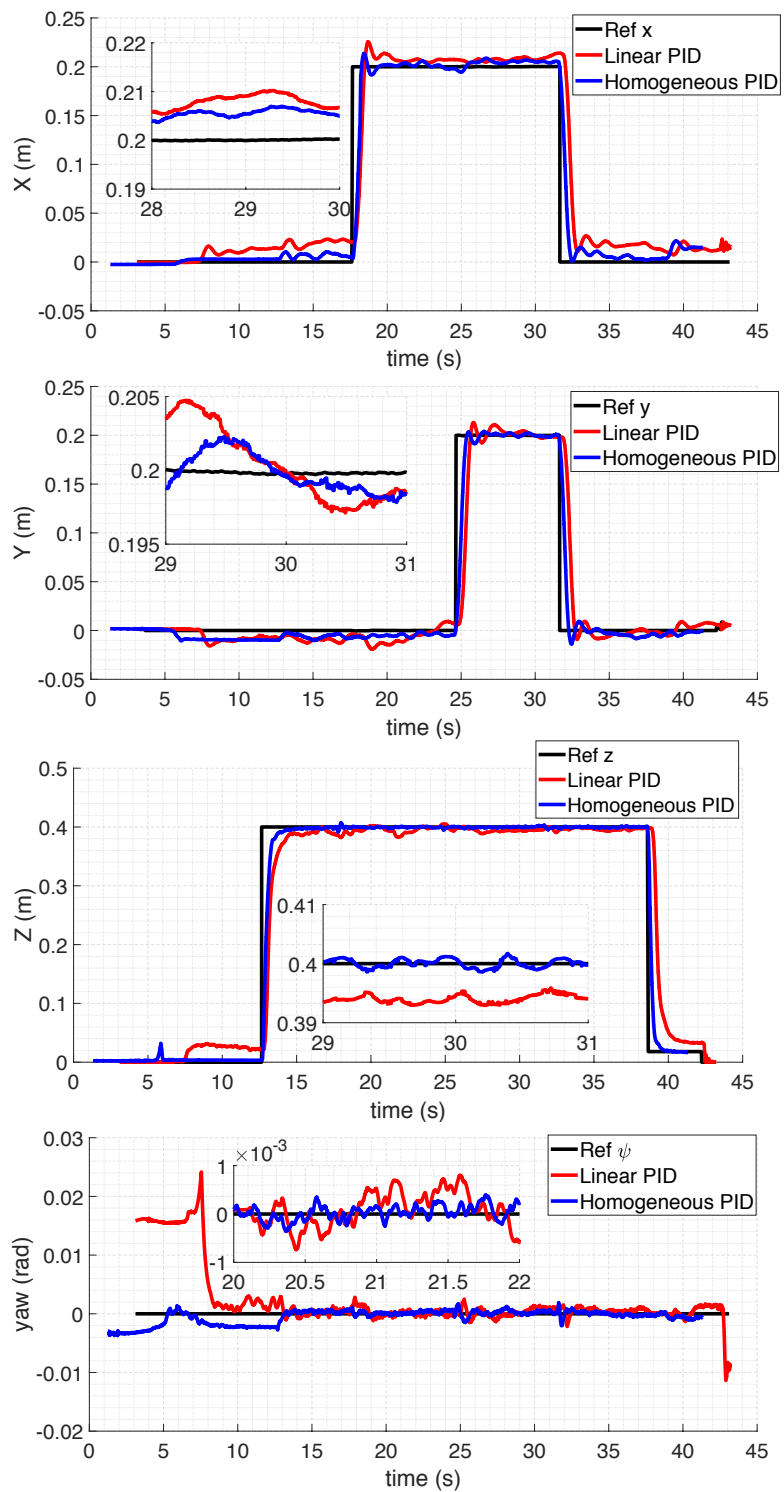

Fig. 2. Quadrotor position tracking comparison in $x, y, z$ and $\psi$

TABLE I

MEAN VALUE OF STABILIZATION ERROR

\begin{tabular}{|c|c|c|c|}
\hline$L_{2}$ Error $(\mathrm{m})$ & Linear & Homogeneous & Improvement \\
\hline \hline$\|$ error $_{x} \|_{L_{2}}$ & 0.0234 & 0.0138 & $41 \%$ \\
\hline$\|$ error $_{y} \|_{L_{2}}$ & 0.0081 & 0.0028 & $66 \%$ \\
\hline$\|$ error $_{z} \|_{L_{2}}$ & 0.0313 & 0.0071 & $77 \%$ \\
\hline$\|$ error $_{\psi} \|_{L_{2}}$ & 0.0036 & 0.0022 & $38 \%$ \\
\hline
\end{tabular}

homogeneous controller proposed in this paper has a faster response and a higher precision.

Table I compares the least square stabilization $\operatorname{errors}\left(L_{2^{-}}\right.$ error) in the steady states. We define that the steady state starts $\approx 2.5 \mathrm{sec}$ after the set-point assignment and ends at the time instant when the new set-point is assigned. The obtained improvement of the $L_{2}$-error is about $40 \%$ and more. The price for this improvement is a bit larger energy consumption. To estimate the energy consumed by 
the controller we use the $L_{2}$ norm of system inputs. The difference between these norms for Quanser PID controller and the homogeneous controller is about $0.5 \%$, i.e., the proposed homogeneous controller consumes only $0.5 \%$ more than the Quanser PID controller, but it can improve at least $40 \%$ precision.

The robustness of the proposed homogeneous controller with respect to the variation of the load is also compared. For this purpose, we added manually an additional load around $0.5 \mathrm{~kg}$ ( $\approx 50 \%$ of the quadrotor's mass) on the body of quadrotor during the flight. The results of the robustness tests for linear PID controller and homogeneous one are presented in Fig. 3 and Fig. 4 respectively. It is clear that the homogeneous PID is more robust with respect to the load variation than the linear PID controller provided by the manufacturer.

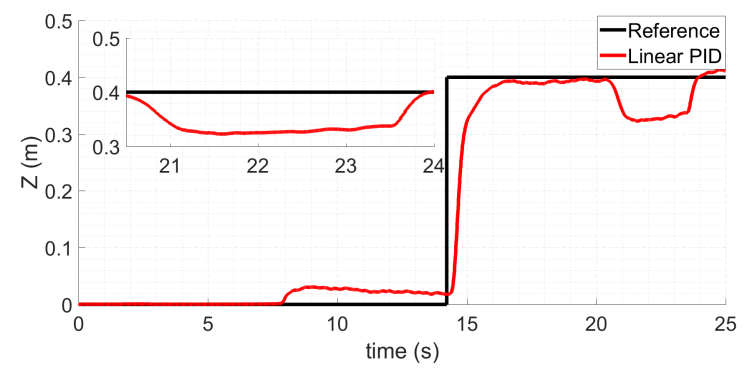

Fig. 3. The response of linear controller to the load disturbance

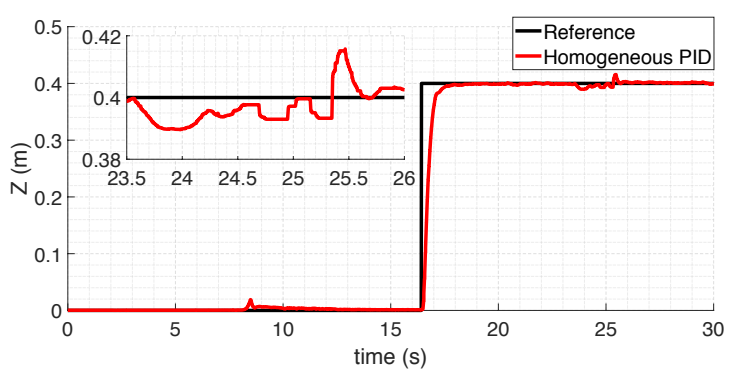

Fig. 4. The response of homogeneous controller to the load disturbance

\section{CONCLUSION}

In this paper, a simple scheme for an "upgrade" of a linear controller to a nonlinear homogeneous one is developed. The nonlinear controller uses the feedback gains of the linear controller and scale them in a generalized homogeneous way dependently of the norm of the system state. The generalized homogeneous systems are known to be more robust and more fast than linear ones. Theoretical results have been supported with real experiments for quadrotor QDrone of Quanser ${ }^{T M}$. The linear controllers provided by the manufacturer have been "upgraded" using the proposed method. The control precision has been improved for more than $40 \%$ by only increasing $0.5 \%$ energy consuming. The robustness with respect to the external disturbance is improved as well. It is worth noting that the methodology for the "upgrade" of the linear controller is rather universal, and it can be utilized for many real control systems.

\section{REFERENCES}

[1] V.I. Zubov. On systems of ordinary differential equations with generalized homogenous right-hand sides. Izvestia vuzov. Mathematica (in Russian)., 1:80-88, 1958.

[2] L. Rosier. Etude de quelques problèmes de stabilization. PhD Thesis, Ecole Normale Superieure de Cachan (France), 1993.

[3] A. Polyakov. Sliding mode control design using canonical homogeneous norm. International Journal of Robust and Nonlinear Control, 29(3):682-701, 2018.

[4] Y. Orlov. Finite time stability and robust control synthesis of uncertain switched systems. SIAM Journal of Control and Optimization, 43(4):1253-1271, 2005.

[5] A. Polyakov, D. Efimov, E. Fridman, and W. Perruquetti. On homogeneous distributed parameters equations. IEEE Transactions on Automatic Control, 61(11):3657-3662, 2016.

[6] V. Andrieu, L. Praly, and A. Astolfi. Homogeneous Approximation, Recursive Observer Design, and Output Feedback. SIAM Journal of Control and Optimization, 47(4):1814-1850, 2008.

[7] M. Kawski. Geometric homogeneity and stabilization. In Arthur Krener and David Mayne, editors, Proc. IFAC Nonlinear Control Symposium, pages 164-169, Lake Tahoe, CA, 1995.

[8] S. P. Bhat and D. S. Bernstein. Geometric homogeneity with applications to finite-time stability. Mathematics of Control, Signals and Systems, 17:101-127, 2005.

[9] E. Bernuau, D. Efimov, W. Perruquetti, and A. Polyakov. On homogeneity and its application in sliding mode control. Journal of The Franklin Institute, 351(4):1866-1901, 2014.

[10] F. Lopez-Ramirez, A. Polyakov, D. Efimov, and W. Perruquetti. Finitetime and fixed-time observer design: Implicit Lyapunov function approach. Automatica, 87(1):52-60, 2018.

[11] H. Nakamura, Y. Yamashita, and H. Nishitani. Smooth lyapunov functions for homogeneous differential inclusions. In Proceedings of the 41st SICE Annual Conference, pages 1974-1979, 2002.

[12] I. Boiko. Non-parametric Tuning og PID Controllers. Springer-Verlag London, 2013.

[13] E.D. Sontag. Nonlinear and Optimal Control Theory, chapter Input to state stability: Basic concepts and results, pages 163-220. SpringerVerlag, Berlin, 2007.

[14] V. I. Utkin. Sliding Modes in Control Optimization. Springer-Verlag, Berlin, 1992.

[15] L.S. Husch. Topological characterization of the dilation and the translation in frechet spaces. Mathematical Annals, 190:1-5, 1970.

[16] V. V. Khomenuk. On systems of ordinary differential equations with generalized homogenous right-hand sides. Izvestia vuzov. Mathematica (in Russian)., 3(22):157-164, 1961.

[17] A. Polyakov, J.-M. Coron, and L. Rosier. On homogeneous finitetime control for linear evolution equation in hilbert space. IEEE Transactions on Automatic Control, 63(9):3143-3150, 2018.

[18] A. Bacciotti and L. Rosier. Lyapunov Functions and Stability in Control Theory. Springer, 2001.

[19] A. Polyakov. Generalized Homogeneity in Systems and Control. Springer, 2020.

[20] K. Zimenko, A. Polyakov, D. Efimov, and W. Perruquetti. Robust feedback stabilization of linear mimo systems using generalized homogenization. IEEE Transactions on Automatic Control, (accepted), 2019.

[21] W. M. Wonham. Linear Multivariable Control: A Geometric Approach. Springer, 1985.

[22] J.-M. Coron and L. Praly. Adding an integrator for the stabilization problem. Systems \& Control Letters, 17(2):89-104, 1991.

[23] J. A. Mercado Uribe, J. A. Moreno, A. Polyakov, and D. Efimov. Integral control design using the implicit lyapunov function approach. In Conference on Decision and Control, 2019.

[24] Samir Bouabdallah and Roland Siegwart. Full control of a quadrotor. In 2007 IEEE/RSJ International Conference on Intelligent Robots and Systems, pages 153-158. Ieee, 2007. 